\title{
Das deutsche Modell auf dem Prüfstand - Innovationen in der Krise
}

Im Zuge der Weltwirtschaftskrise verändern sich auch die globalen Marktverhältnisse. Die Frage, ob Deutschland als Gewinner aus der Krise hervorgeht, kann heute noch nicht beantwortet werden. Ausschlaggebend wird sein, ob die deutschen Unternehmen erfolgreich ihre Innovationsfähigkeit weiterentwickeln. Könnte die Mitbestimmung einen Beitrag dazu leisten, das Wissen sowie die Kooperation der Beschäftigten zu aktivieren und besser in den Innovationsprozess einzubringen?

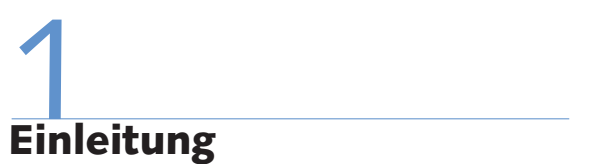

In der aktuellen dramatischen Wirtschaftskrise wird die Frage nach der Wettbewerbsund Zukunftsfähigkeit des deutschen Modells in aller Schärfe aufgeworfen - werden doch auf den Weltmärkten die Karten neu gemischt. Die Schwellenländer holen auf, insbesondere China verfolgt eine dezidierte High-Road-Strategie, die den alteingesessenen Industrieländern ihre Dominanz auf den Märkten für höherwertige Produkte bereits jetzt oder in absehbarer Zukunft streitig macht (Xielin et al. 2007). Durch die Krise werden sich die Kräfteverhältnisse auf dem Weltmarkt beschleunigt verändern.

Wie kann sich die deutsche Volkswirtschaft gegen den wachsenden Konkurrenzdruck behaupten? Dies ist die zentrale Frage, die uns in den kommenden Jahren beschäftigen wird. Die Antwort hierauf ist evident: Zur Sicherung der eigenen Wettbewerbsvorteile gegenüber der internationalen Konkurrenz ist die beschleunigte Einführung und Umsetzung von Innovationen nötig. Dies ist allerdings kein leichtes Unterfangen, denn technologische Vorsprünge werden in einer globalisierten Wirtschaft immer schneller aufgeholt.

Regierungen formulieren weltweit Strategien, die Innovationsprozesse beschleunigen und auf der Ebene der Volkswirtschaft hierfür die Voraussetzungen schaffen sollen. Verstärkte finanzielle Anstrengungen im Bildungssektor, in der staatlichen Forschung sowie im Bereich Forschung und
Entwicklung (F\&E) der Unternehmen sind hierbei nur ein Element. Veränderungen im institutionellen Arrangement, z. B. in der Verzahnung staatlicher und privater Forschungseinrichtungen, in der Ausrichtung des Finanzsektors auf eine gezielte und großzügigere Bereitstellung von Risikokapital sowie in der Intensivierung der horizontalen und vertikalen Kooperation von Unternehmen, werden ebenfalls verfolgt.

Mehr oder weniger bemühen sich alle wichtigen Volkswirtschaften um einen solchen strategischen Ansatz - mit einer je spezifischen Ausprägung des Verhältnisses von Markt und staatlicher Politik. In einem Fall kommt dem Staat mehr Gewicht zu wie z. B. in China -, in anderen Fällen wird das freie Spiel der Marktkräfte eher präferiert - beispielsweise in den USA (Gerlach/ Ziegler 2007).

Welche Karten hat Deutschland in diesem Spiel? Das vorliegende Schwerpunktheft der WSI-Mitteilungen fragt, ob das deutsche Mitbestimmungsmodell ein „Pfund“ im globalen Innovationswettbewerb ist, ob es Innovationsprozesse eher behindert oder selbst als quantité négligeable zu betrachten ist. Die folgenden Beiträge nähern sich in erster Linie empirisch auf der betrieblichen Ebene dieser Frage. Ihren Ergebnissen soll nicht vorgegriffen werden. Ziel des einleitenden Beitrags ist vielmehr, eine geraffte, notwendigerweise selektive Darstellung des Forschungsstandes zum Themenfeld „Innovation und Mitbestimmung “ zu geben. Zum einen soll vorweg eine Standortbestimmung vorgenommen werden, die den Ausgangspunkt für eine Innovationsoffensive markiert (Abschnitt 2 und 3), zum anderen wird das deutsche Innovationssystem umrissen, in dem innovationspolitische Strategien umgesetzt werden (Abschnitt 4 und 5). Das Fazit richtet den Blick auf Wege zur Durchsetzung einer mitbestimmungsfreundlichen Sicht von Innovationsprozessen (Abschnitt 6).

\section{Standortbestimmung des deutschen Innovations- modells}

Mit dem Verweis auf die hohen Exportüberschüsse wurde in der Vergangenheit die Frage nach der anhaltenden Konkurrenzfähigkeit des deutschen Innovationsmodells regelmäßig positiv beantwortet. Das Hochlohnland Deutschland agiert mit hochwertigen Produkten - häufig verknüpft mit produktbezogenen Dienstleistungen - erfolgreich auf den Weltmärkten. Die Liste der „hidden Champions“ ist lang. So haben nach Angaben des Verbandes der deutschen Maschinen- und Anlagenbauer

Frank Gerlach, Dr., Ökonom, war bis 2009 Leiter des Referats "Strukturwandel Innovationen und Beschäftigung " in der Abteilung Forschungsförderung der HansBöckler-Stiftung. e-mail: Frank-Gerlach@boeckler.de Astrid Ziegler, Dr., Ökonomin, ist Leiterin des Ressorts Industriepolitik beim Vorstand der IG Metall, Frankfurt/Main. e-mail: Astrid.Zieg/er@igmetall.de 
in 18 von 30 international vergleichbaren Maschinengruppen der Branche die deutschen Hersteller bei den Ausfuhren die Nase vorn (VDMA 2009, S. 19). Für die Wettbewerbsfähigkeit einer Volkswirtschaft ist die Industrie nach wie vor entscheidend, da in ihr die meisten Innovationen erzeugt bzw. in Produkte umgesetzt werden. $\mathrm{Zu}$ dem sind viele, insbesondere hochwertige Dienstleistungen auf die industrielle Produktion bezogen bzw. mit ihr eng verbunden (Kalmbach et al. 2005).

Trotz auf den Weltmärkten gut positionierter deutscher Unternehmen zeigt ein Blick auf die Strukturen der Industrie nach den vorliegenden Untersuchungen ein gemischtes Bild. Deutschland ist insbesondere stark im Bereich der nach Definition der $\operatorname{OECD}(2002 ; 2005)$ hochwertigen Technologien, d. h. der Branchen mit einer F\&EIntensität von $3 \%$ bis $5 \%$. Hier sind speziell der Maschinenbau und die Automobilindustrie, aber auch die chemische Industrie und die Elektroindustrie zu nennen (Licht et al. 2009). Auf diese Sektoren konzentrieren sich die Exportüberschüsse. Zudem ist in ihnen deutlich mehr als ein Drittel der Beschäftigten des verarbeitenden Gewerbes beschäftigt. Allerdings birgt die starke Konzentration auf einige exportstarke Branchen erhebliche Gefahren. Die Krise der Automobilindustrie und des Maschinenbaus hat die Fragilität des deutschen Exportmodells in den letzten zwei Jahren offengelegt.

Demgegenüber ist Deutschland im internationalen Vergleich im Bereich der Spitzentechnologiebranchen, d.h. der Branchen mit einer F\&E-Intensität von über $5 \%$, schwächer vertreten. Zwar hat ihre Bedeutung in den letzten Jahren - gemessen an dem Anteil an der gesamten Wertschöpfung des verarbeitenden Gewerbes - von $8,9 \%$ (1995) auf $12 \%$ (2006) zugenommen, jedoch stagniert ihr Anteil an den gesamten Exporten bei etwa $15 \%$ (Licht et al. 2009). Im Bereich Informations- und Kommunikationstechnologie (IuK), einer für die Hochtechnologie nach wie vor zentralen Branche, ist Deutschland bis auf SAP und Infineon eher schwach vertreten. $\mathrm{Zu}$ Recht stellt der Bericht zur technologischen Leistungsfähigkeit Deutschlands fest: „IuKund Medientechnik waren nie eine Spezialität Deutschlands. Deutschland ist dort stark auf Importe angewiesen; mit zunehmender Bedeutung dieser Technologien "belastet" dies die Außenhandelsbilanz von Jahr zu Jahr stärker“ (BMBF 2006, S.27).
Ein weiteres Beispiel ist die Pharmaindustrie, die im internationalen Vergleich deutlich an Boden verloren hat (Jürgens/Sablowski 2008 S. 19ff; Gaisser et al. 2005).

Die Steigerung der privaten und öffentlichen F\&E-Ausgaben für Spitzentechnologien in den letzten Jahren hat den in einigen Bereichen bestehenden Rückstand Deutschlands gegenüber anderen Ländern nur geringfügig reduzieren können. Andererseits nimmt die Bundesrepublik bei bestimmten Spitzentechnologien wie der Nano- und der Biotechnologie wie auch der Umwelttechnologie eine führende Position ein.

Die Berichte des Bundesministeriums für Bildung und Forschung (BMBF) zur technologischen Leistungsfähigkeit Deutschlands zeichnen ebenfalls ein gemischtes Bild (BMBF 2006). Im internationalen Vergleich steht Deutschland recht gut da, allerdings ist seine Position aufgrund der schmaler gewordenen Wettbewerbsfähigkeit heute fragiler - wie die jüngste Krise gezeigt hat. Noch immer hat die Bundesrepublik das Lissabon-Ziel, die Verwendung von $3 \%$ des Bruttoinlandsproduktes für F\&E, nicht erreicht. 2009 werden es etwa 2,6\% sein, 2010 ist sogar mit einem prozentualen Rückgang zu rechnen. Jedoch hat die Bundesrepublik keineswegs, wie manchmal unterstellt wird, ihre Stärken lediglich in ausgereiften Technologien mittleren Niveaus. Gerade in ökologisch orientierten Technologien, die sich von neuen Formen der Energiegewinnung bis zu energieeffizienten Verfahren bzw. Maschinen erstrecken, ist Deutschland z. T. Weltmarktführer. Allerdings ist nicht zu leugnen, dass andere Staaten, insbesondere die asiatischen, in den letzten Jahren erhebliche Anstrengungen unternommen haben, um aufzuholen bzw. zu überholen. Beispielsweise ist Korea - nach dem Indikator F\&E-Ausgaben - Forschungsspitzenreiter sogar noch vor Japan und den skandinavischen Ländern. China hat in dem zurückliegenden Jahrzehnt seine Ausgaben für Forschung und Entwicklung vervierfacht. Das Land investiert gewaltige Summen in diesen Bereich und erreicht heute schon 1,5 $\%$ des Bruttoinlandsprodukts. Die Konjunkturprogramme des vergangenen Jahres wurden für eine weitere Erhöhung der F\&E-Ausgaben genutzt. Mittelfristig droht somit ein massiver Einbruch in Domänen der deutschen Industrie. Ein Blick auf den sich zuspitzenden Wettbewerb im Automobilbereich zeigt dies nur zu deutlich.

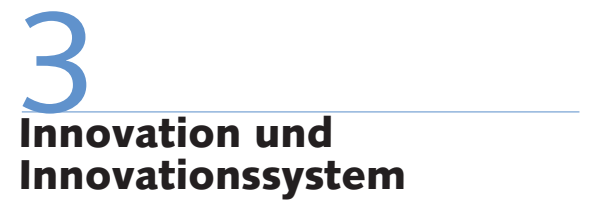

Unter Innovationen werden in diesem Beitrag betriebliche Neuerungen und Veränderungen in Unternehmen verstanden. Sie sind eingebettet in ein Innovationssystem, das national, regional oder sektoral analysiert werden kann. Innovationssysteme umfassen nach international akzeptiertem Verständnis die „Kulturlandschaft“ all jener Institutionen, die wissenschaftlich forschen, Wissen akkumulieren und vermitteln, die Arbeitskräfte ausbilden, die Technologien entwickeln, die innovative Produkte und Verfahren hervorbringen sowie verbreiten (Grupp et al. 2004, S. 87f.). Die Unternehmen sind ein Element dieses Systems, wobei sie mit den anderen Institutionen interaktive Beziehungen unterhalten (können).

Die Generierung und Umsetzung von Innovationen wird in der Innovationsforschung nicht mehr als linearer Prozess verstanden. Während man früher von einer Kette von Forschung, Entwicklung bis hin zum Markteintritt der Neuerungen ausging, hat sich mittlerweile ein systemisches Verständnis von Innovationen durchgesetzt, das Netzwerke zugrunde legt, in denen Innovationen entstehen und realisiert werden. Es sind nicht allein die Marktteilnehmer, die durch Kooperation oder Interaktion Innovationen auf den Weg bringen, sondern solche Netzwerke reichen weit über Marktbeziehungen hinaus. Hierauf haben zu Recht Autoren wie Lundvall (1992), Nelson (1993) und Dreher (2008) hingewiesen. Insbesondere in der Phase der Umsetzung von Inventionen, also beim Schritt von der bloßen Erfindung zu Innovationen, spielen Kapitalmarktbeziehungen, komplexe Wissensarchitekturen, Arbeitsbeziehungen, neue Geschäftsmodelle und spezifische Aneignungsbedingungen von neuem Wissen eine Rolle. Zugleich sind in Innovationsprozessen Rückkopplungsschleifen „eingebaut“ bzw. erforderlich, die Korrekturen ermöglichen und wichtige Voraussetzungen für erfolgreiche Innovationen auf der betrieblichen Ebene sind. Für die Realisierung von Innovationen auf der Ebene des Unternehmens wird ein Innovationsmanagement im Sinne einer umfassenden und zielgerichteten Aktivität für erforderlich gehalten (Gerybadze 
2004). Gerybadze (ebd.) und Tidd et al. (2001) entwickelten hier das Konzept der "flexiblen Routinen“, das eingespielte Innovationswege und Lernmöglichkeiten verknüpft. Eine wichtige Voraussetzung ist allerdings eine entsprechende Wahrnehmungs- und Kommunikationsbereitschaft.

Als Indikatoren für einen Innovationserfolg werden gewöhnlich die Entwicklung der Umsätze, des Marktanteils, der Kosten, der Produktivität sowie der Beschäftigung ausgewiesen. Allerdings greift eine Beschränkung des Erfolges allein auf wirtschaftliche Aspekte zu kurz, da das Innovationsumfeld dabei ausgeklammert wird. Bessere Arbeitsbedingungen sowie eine anspruchsvollere Arbeit im Sinne des Konzeptes "gute Arbeit" sollten mit Blick auf ein systemisches Verständniss in ein Bezugssystem für die Bewertung von Innovationen einbezogen werden. Auch diese wirken sich im Endeffekt auf die Entwicklung der genannten engeren wirtschaftlichen Indikatoren aus.

Zudem stellt sich die Frage, ob das Kriterium des Markterfolges für eine Bewertung von Innovationen auf der Ebene des Unternehmens allein ausreicht. Wenn die unternehmensinternen wie -externen Anreizsysteme falsch konstruiert sind, kann es $\mathrm{zu}$ einer übertriebenen Risikobereitschaft und zu übermäßig kurzfristigem Verhalten kommen. Ökologische, ökonomische und soziale Kosten werden von den Unternehmen soweit möglich und erlaubt gern externalisiert - mit häufig desaströsen Konsequenzen. Während z. B. der Nutzen (nicht die Profitabilität) vieler neuer Finanzprodukte, die in den vergangenen Jahren als innovativ angepriesen wurden, schwer nachzuweisen, wenn überhaupt zu beziffern ist, so sind die damit verbundenen Kosten offenkundig und enorm (Stiglitz 2009). Unvollkommene Märkte - zu erklären u. a. mit „Animal Spirits“ (Akerlof/ Shiller 2009) - führen eben häufig zu anderen Ergebnissen als es von „Marktpuristen" für möglich gehalten wird.

Ein Innovationsverständnis, das primär auf die technologischen Produktinnovationen abhebt - etwa im Sinne des OsloManual (OECD 2005) - greift deshalb zu kurz, weil die Einbettung von Innovationen in ein soziales System aus dem Blickfeld zu geraten droht. Bereits Schumpeter (1932) hat darauf hingewiesen, dass Innovationen nicht nur neue Produkte, sondern auch Veränderungen von Dienstleistungen, technischen Prozessen und Organisationsstrukturen sowie die Erschließung neuer Bezugsquellen oder Vorleistungen umfassen. Ein ganzheitliches Innovationsverständnis hat diese vier Aspekte zu berücksichtigen und damit technische Entwicklungen im engeren Sinne mit sozial-ökonomischen Veränderungen, wie z. B. im Bereich von Organisations- und Führungsstrukturen, von Arbeitszeitmodellen und betrieblicher Qualifizierung, also Maßnahmen und Aktivitäten, die schwerpunktmäßig der Arbeitspolitik zugeschrieben werden können, zu verbinden.

Internationale Vergleiche von Innovationsfähigkeit konzentrieren sich jedoch in der Regel auf technische Produktinnovationen und werden häufig - auch aus Gründen empirischer Machbarkeit - mithilfe quantitativer Indikatoren wie z. B. der F\&E-Intensität oder der Patentstatistiken durchgeführt. Dies ist zwar im wissenschaftlichen Sinne wichtig, reicht aber keineswegs aus. Andere qualitative Dimensionen, auf die Arreger (1976) bereits früh hingewiesen hat, geraten dadurch aus dem Blickfeld. Soziale Innovationen - etwa im Bereich der Mobilität bei der Umsetzung eines integrierten Verkehrskonzeptes können für die Lösung von Problemen wie der Erderwärmung ebenso wichtig sein wie Produktinnovationen - z. B. die Entwicklung neuer Antriebssysteme.

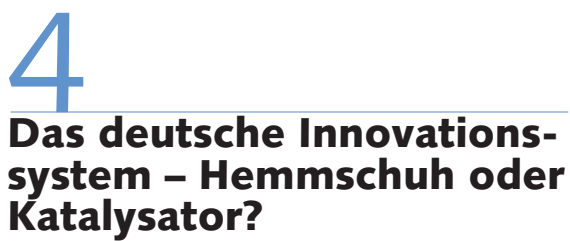

Einen großen Einfluss auf die wissenschaftliche wie politische Diskussion zu den Stärken und Schwächen des deutschen Innovationssystems hatte die grundlegende Arbeit von Peter Hall und David Soskice (2001). Danach ist es kein Zufall, dass Deutschland Schwächen auf den Feldern der hochwertigen und Stärken bei ausgereiften Technologien aufweist. Hierdurch würden sich die Wachstumsunterschiede zwischen Volkswirtschaften wie den USA und Deutschland erklären. Für Hall/Soskice beruht die technologische Spezialisierung auf einer je spezifischen Ausprägung des jeweiligen nationalen Institutionensystems, das durch einen breiten Set aufeinander bezogener und zueinander „passender" Einrichtungen charakterisiert sei.
Idealtypisch sei zwischen zwei Spielarten des Kapitalismus zu unterscheiden: In liberalen Marktwirtschaften erfolge die Koordination des Handelns zwischen den Unternehmen und anderen Akteuren primär über den Markt, koordinierte Marktwirtschaften seien stärker durch nicht-marktmäßige Beziehungen charakterisiert. Prototypisch für die erste Gruppe seien die USA, für die zweite die Bundesrepublik Deutschland.

Ein wichtiges Element des Institutionensystems seien die Arbeitsbeziehungen, die (mit)verantwortlich für die Innovationsschwäche Deutschlands im Bereich der radikalen Innovationen seien. So gesehen ist das deutsche Modell der Mitbestimmung nicht durchweg ein Pluspunkt im internationalen Innovationswettbewerb.

Der Erklärungsansatz von Hall/Soskice schien plausibel zu sein, vermochte er doch spezifische Stärken der US-Wirtschaft etwa im Bereich der IT-Technologien zu begründen, die durch den Hinweis auf das Silicon Valley und die „Roaring Nineties" (Krueger/Solow 2001; kritisch Stieglitz 2004) unterstrichen wurden. Dass andererseits die deutsche Volkswirtschaft in dem seit etwa 1990 „laufenden“ Zyklus der Informationstechnik mit Digitalisierung, Mobiltelefon und Internet - wie bereits erwähnt - bestenfalls in der zweiten Reihe steht, schien ein weiterer Beleg für unterschiedliche Innovationspotenziale - je nach Spielart des Kapitalismus zu sein.

Dennoch wurde der Analyse von Hall/ Soskice widersprochen. Die Schwierigkeiten, radikale und inkrementelle Innovationen definitorisch voneinander abzugrenzen, sowie die schmale Datenbasis - die Autoren vergleichen im Grunde nur die Patentspezialisierung Deutschlands und der USA - wurden als Einwände gegen die Tragfähigkeit der These vorgebracht (Jürgens/Sablowski 2008, S. 112ff.). Zudem scheinen koordinierte Volkswirtschaften in ihrer Pfadabhängigkeit nicht so eindeutig auf inkrementelle Innovationen festgelegt zu sein wie Hall/Soskice suggerieren: Skandinavische Länder sind wettbewerbsfähig in bestimmten Segmenten des IT-Bereichs, und die Bundesrepublik ist führend in der Nanotechnologie und hat in den Biotechnologien deutlich aufgeholt.

Auf einen weiteren wichtigen Punkt in der Diskussion um Spielarten des Kapitalismus haben Jürgens/Sablowski (2008, 14f.) 
hingewiesen. Im Zeitalter der Globalisierung könne nicht unbedingt davon ausgegangen werden, dass Innovationssysteme national sein müssen. Für Hall/Soskice seien nationale Innovationssysteme die unabhängige Variable, während die Lokalisierung von Branchen, von Forschungsund Produktionsprozessen abhängige Variablen seien. Damit werde aber von den Besonderheiten sektoraler, lokaler und auch betrieblicher Innovationsprozesse abstrahiert, deren Verlauf im Zusammenspiel verschiedener Institutionen, unter ihnen eben auch der Mitbestimmungseinrichtungen, empirisch genauer zu analysieren sei.

Spätestens seitdem im Kontext der von den USA ausgehenden Finanz- und Weltwirtschaftskrise die massiven Strukturschwächen der US-Wirtschaft deutlich geworden sind, wird das US-amerikanische Innovationssystem skeptischer beurteilt. Paul Volcker (2009), ehemaliger Vorsitzender der US-Notenbank Federal Reserve, forderte unlängst eine Re-Industrialisierung der USA und kritisierte, dass die Vereinigten Staaten in der Zukunftsbranche der erneuerbaren Energien in der Forschung und Entwicklung weit vorn seien, aber hergestellt werde diese Technik in Deutschland und von dort in den Rest der Welt verkauft - also ein Entwicklungsmuster, das in der Vergangenheit häufig Deutschland vorgehalten worden ist. ${ }^{1}$

Im Grunde ist damit eine Diskussion aufgegriffen worden, die in den 1980er Jahren u.a durch die MIT-Kommission mit empirischen Branchenstudien zum Niedergang der US-Industrie (mit)angestoßen wurde (Dertouzos et al. 1990). ${ }^{2}$ In den "Roaring Nineties" geriet das in Vergessenheit. Die Autoren kritisierten damals die geringe Innovationskraft der US-Unternehmen und führten sie $u$. a. auf eine mangelhafte betriebsinterne Kooperation zwischen Belegschaft und Management, fehlendes Teamwork zwischen den verschiedenen betrieblichen Abteilungen sowie fehlende längerfristige, auf gegenseitigem Vertrauen basierende Kooperation mit Lieferanten und Kunden zurück. Nicht ausreichend war nach Ansicht der Kommission ebenfalls die horizontale Kooperation zwischen Unternehmen, die gleiche bzw. ähnliche Produkte herstellen. In diesen Bereichen liegen nun - hierauf weist Ralf Reichwald (in diesem Heft) zu Recht hin - spezifische Stärken der deutschen Schlüsselbranchen.
Die Frage, ob das deutsche Innovationssystem Innovationen fördert oder behindert und sich insbesondere als Hemmschuh für radikale Innovationen erweist, sollte empirisch unter Einbeziehung der Branchen- und Unternehmensebene untersucht werden. Die Analyse von nationalen Innovationssystemen reicht offensichtlich nicht aus, um schlüssige Antworten geben zu können.

\section{5 \\ Innovation und Mitbe- stimmung - ein Spannungs- verhältnis?}

\subsection{PRO- UND KONTRA-ARGUMENTE}

Mitbestimmung ist ein zentrales Element des deutschen Produktionssystems mit seinen charakteristischen Innovationsmustern. In der Diskussion über ihre Auswirkungen auf die Innovationsfähigkeit von Unternehmen wird nun kritisch auf längere Entscheidungswege und geringere Flexibilität in den Betrieben hingewiesen. Wenn negative Konsequenzen für die Beschäftigung befürchtet werden, dann seien Blockaden gegen die Einführung von Prozessinnovationen vonseiten der Träger der Mitbestimmung möglich. Dies könne z. B. die Umsetzung von Produktinnovationen behindern, da diese häufig mit der Reorganisation von Produktionsprozessen verbunden sei. ${ }^{3}$

Dem wird entgegengehalten, dass die Mitbestimmung den Informationsfluss verbessere und sich hierdurch positive Auswirkungen auf den technischen Fortschritt ergäben. Zudem würden - wenn etwa in den Aufsichtsräten Entscheidungen gemeinsam gefällt werden - diese von den Beschäftigten eher akzeptiert und als fair empfunden. Hierdurch könne sich die Produktivität erhöhen. Die Mitbestimmung ermögliche somit eine bessere Steuerung des Innovationsprozesses durch die Berücksichtigung der Interessen beider Unternehmensparteien. Des Weiteren wird darauf verwiesen, dass die Mitbestimmungsträger verhindern können, dass Manager ihre eigenen Interessen, die nicht zwangsläufig den Interessen des Unternehmens entsprechen müssen, verfolgen. Sie verfügten häufig über bessere Informationen als die Kapitaleigner und könnten somit rascher auf Fehlentwicklungen im Unternehmen reagieren.
Solche Argumente, die die positiven Auswirkungen der Mitbestimmung auf die Innovationsfähigkeit unterstreichen, negiert der Property-Rights-Ansatz. Danach verfolgen Arbeitnehmer und ihre Vertreter in Betriebs- und Aufsichtsräten aus strukturellen Gründen eher kurzfristige Ziele anders als die Kapitaleigner (Furubotn 1978; Alchian 1984). Langfristig orientierte Investitionen seien deshalb nicht im Interesse der Arbeitnehmer. Dies führe zu Innovationsdefiziten und zu einer Überbetonung der Beschäftigungssicherung. Mitbestimmung teile die Entscheidungsrechte im Unternehmen und reduziere damit die Entscheidungsfähigkeit und -bereitschaft. Dem wird jedoch entgegengehalten, dass sich Unternehmensstrategien häufig an den kurzfristigen Interessen der Shareholder orientieren und daher die Annahme eines strukturell kurzfristigeren Zeithorizonts der Arbeitnehmer als sehr fragwürdig anzusehen sei.

Angesichts der Pro- und Kontra-Argumente ist es letztlich eine empirisch zu beantwortende Frage, in welcher Weise sich die Mitbestimmung auf die Realisierung von Innovationsprozessen auswirkt. $\mathrm{Zu}$ differenzieren ist hierbei nach dem Betriebsverfassungsgesetz und dem Mitbestimmungsgesetz. Betriebsräte haben lediglich Informationsrechte bezüglich Innovations- bzw. Investitionsvorhaben und können Innovationsvorschläge zur Beschäftigungssicherung auf Basis des $\$ 92 \mathrm{a}$ BetrVG einbringen. Demgegenüber sind die Mitbestimmungsrechte der Arbeitnehmervertreter in den Aufsichtsräten umfassender. Die „zustimmungspflichtigen Geschäfte" betreffen Entscheidungen, die sich auf die gegenwärtige Situation des Unternehmens wie auch auf strategische Festlegungen beziehen. Die Planungen von In-

\footnotetext{
Diese Kritik ist nur ein Beispiel für die zurzeit in den USA breit geführte Diskussion.

2 Keineswegs standen die Autoren mit ihrer Kritik allein. Bereits in der ersten Hälfte der 1980er Jahre analysierten Bluestone/Harrison (1982) sowie Bowles et al. (1984) den vermeintlichen Niedergang der US-Wirtschaft. In der Euphorie der 1990er Jahre war davon keine Rede mehr, obwohl die Innovations- und Wettbewerbsfähigkeit in vielen Branchen zu gering blieb - wie die anhaltenden Zahlungsbilanzprobleme der USA zeigten. Erst in der "Katerstimmung “ der aktuellen Krise wird wieder der Finger in die Wunde gelegt.

3 Schließlich sind die negativen Wirkungen von Prozessinnovationen im Vergleich zu Produktinnovationen auf Arbeitsplätze und Beschäftigte größer (vgl. Ziegler in diesem Heft).
} 
novationen sind hierin einbezogen. Jürgens/Lippert (2005) weisen zu Recht darauf hin, dass Aufsichtsräte Innovationsvorhaben nicht nur billigen oder ablehnen, sondern auch anregen können. Da zudem in den Aufsichtsräten Entscheidungen überwiegend einstimmig gefällt werden und somit für alle Mitglieder akzeptabel sind, kann der Einfluss der Arbeitnehmervertreter auf die Innovationsstrategie des Unternehmens beträchtlich sein. Aber auch die Betriebsräte haben - trotz eingeschränkter Rechte - in der Praxis die Möglichkeit, z. B. über die Mitbestimmung in Personalangelegenheiten sowie durch eine geschickte Informationspolitik die Innovationspolitik des Unternehmens mitzubestimmen.

\subsection{EMPIRISCHE EVIDENZ}

Im Folgenden werden die Ergebnisse empirischer Studien in aller Kürze vorgestellt: Eine neue Untersuchung von Kraft et al. (2009) vergleicht die Auswirkungen des Mitbestimmungsgesetzes von 1976 auf die Innovationsfähigkeit von Unternehmen. Hierzu wurden mitbestimmte Aktiengesellschaften in den Zeiträumen von 19711976 und 1981-1990 mit nicht-mitbestimmten Aktiengesellschaften verglichen. Intervenierende Variablen wurden im ökonometrischen Modell kontrolliert. Als Indikator für Innovation diente die Anzahl der Patente. Die Ergebnisse der Untersuchung sind recht eindeutig: Die Hypothese, dass Mitbestimmung Innovationen behindere, konnte nicht bestätigt werden. Wenn Mitbestimmung tatsächlich zu einer Verzögerung von Entscheidungen führen sollte, dann müsse es Gegeneffekte - wie z. B. einen besseren Informationsfluss, eine effektivere Kontrolle von eigeninteressierten Managern - geben. Eher könne eine positive Wirkung auf die Innovationsfähigkeit konstatiert werden. Danach betrachten Arbeitnehmervertreter in Aufsichtsräten langfristig wirksame Investitionen in "Wissenskapital“ als eine Chance, Arbeitsplätze zu sichern.

Freilich ist einzuräumen, dass die Aussagekraft der Studie insofern begrenzt ist, als die Wirkung von Patenten auf die Profitabilität und damit auf das Wachstum von Unternehmen bestritten wird: Es ist bekannt, dass viele Innovationen nicht zu Patenten führen. Manche Erfinder scheuen die Kosten des Patentierens und versuchen deshalb, ihre Erfindung auf anderen Wegen „zu schützen“. Darüber hinaus ist es - an- gesichts der Komplexität des Innovationsprozesses - immer gewagt, Innovationen mit nur einem Indikator zu messen. Für quantitative Untersuchungen sollten deshalb weitere Indikatoren einbezogen werden. Außerdem sind qualitativ orientierte Fallstudien, die typologisch die Rolle der Arbeitnehmervertreter in Innovationsprozessen genauer aufschlüsseln können, erforderlich. ${ }^{4}$

Andere quantitative empirische Analysen zum Einfluss des Betriebsrates auf die Innovations- und Investitionstätigkeit ergeben kein einheitliches Bild. ${ }^{5}$ Es gibt Studien, die keine Wirkungen des Betriebsrates auf Prozess- und Produktinnovationen ausmachen - wenn der Betriebsrat als exogene Determinante in Form einer Dummyvariablen eingeht (Addison et al. 2001; Dilger 2002; Jirjahn 1998). Blume/Gerstlberger (2007) bestätigen dieses Ergebnis. Allerdings begünstige die Existenz von Betriebsräten Formen von Partizipation im Arbeitsprozess sowie im innerbetrieblichen Wissensmanagement, wodurch sich wiederum indirekt ein positiver Einfluss auf die Forschungs- und Entwicklungsaktivitäten in den Unternehmen ergäbe.

Wird die Betriebsratsvariable hingegen instrumentiert in die Regression eingeführt, also ein alternatives statistisches Schätzverfahren genutzt, so erhöht sich bei seiner Existenz die Wahrscheinlichkeit von Produktinnovationen (Addison et al. 1996). Eindeutiger scheint der Zusammenhang bei Umweltinvestitionen zu sein (Askildson et al. 2002). Danach lässt sich ein positiver Effekt des Betriebsrates ausmachen, der sich durchaus plausibel begründen lässt. Danach haben Beschäftigte ein unmittelbares Interesse, eine Umweltverschmutzung am Arbeitsplatz sowie in der Umgebung des Werkes niedrig zu halten. In der Regel werden die Betriebsräte diese Interessen aufgreifen und sich für Investitionen, die die Umweltverschmutzung reduzieren, einsetzen.

$\mathrm{Zu}$ ganz anderen Resultaten kommen FitzRoy/Kraft (1990) im Rahmen einer Untersuchung mit einer relativ kleinen Stichprobe. Danach ist der Umsatzanteil neuer Produkte in mitbestimmten Betrieben und bei hohem gewerkschaftlichen Organisationsgrad signifikant niedriger als in anderen Betrieben.

Hingegen sind die Ergebnisse bei der Produktivität, dem Umsatz und der Wertschöpfung eindeutiger. Die Mehrzahl der empirischen Untersuchungen konstatiert hier einen positiven Zusammenhang wenn nicht getrennt für verschiedene Betriebsgrößen analysiert wird. Ausgelöst werden können diese Effekte durch ein positives Betriebsklima, aber auch durch eine verstärkte direkte Partizipation. Indirekt „profitiert“ die Innovationsfähigkeit des Unternehmens von diesem positiven $\mathrm{Zu}$ sammenhang, wenn hierdurch die Akzep$\operatorname{tanz}$ von Investitionsentscheidungen erhöht wird. Des Weiteren begünstigt die betriebliche Mitbestimmung die betriebliche Weiterbildung sowie bestimmte Arten betrieblicher Reorganisationsmaßnahmen - wie z. B. die Gruppenarbeit (Gerlach/ Jirjahn 1998; Hübler/Jirjahn 2002). Beides sind wichtige Elemente in einem betrieblichen Innovationsprozess. Hingegen besteht zwischen der Verlagerung von Verantwortung auf untere Hierarchieebenen und Betriebsräten ein negativer Zusammenhang (Hübler/Jirjahn 2002). Möglicherweise befürchten Betriebsräte hierdurch eine Schwächung ihrer Position. Dieses hätte negative Auswirkungen auf die Produktivität und möglicherweise auch auf die Innovationsbereitschaft.

Die Effekte der betrieblichen Mitbestimmung in kleinen und mittelgroßen Betrieben scheinen generell recht gering $\mathrm{zu}$ sein. In einem beträchtlichen Teil der Betriebe dieses Typs gibt es keinen Betriebsrat. Dies gilt insbesondere für die Start-ups, die in neuen Bereichen wie z. B. der Biotechnologie oder der Nanotechnologie häufig Innovationsträger sind. Effekte der Mitbestimmung gibt es in diesen Unternehmen nicht - im Übrigen ein weiteres Argument gegen die angeblich mitbestimmungsbedingte Schwäche der deutschen Wirtschaft bei radikalen Innovationen. In den Betrieben dieser Größenordnung lässt sich allenfalls ein indirekter Effekt auf die Wirksamkeit von Innovationen durch die empirisch gesicherten positiven Wirkungen des Betriebsrates auf abteilungsinterne

\footnotetext{
4 Das noch nicht abgeschlossene, von der HansBöckler-Stiftung geförderte Projekt von Jürgens und Lippert zum Thema "Corporate Governance, Arbeitnehmerbeteiligung und Innovation. Eine empirische Untersuchung in der deutschen, schwedischen und US-amerikanischen Automobilindustrie" wird hier möglicherweise auf der Basis von Fallstudien erste Antworten geben können.

5 Im Folgenden orientieren wir uns an dem Überblick von Hübler (2003) über die empirischen Untersuchungen zur Rolle des Betriebsrates bei der Unternehmensentwicklung sowie an einigen neueren Studien.
} 
Reorganisations- sowie Weiterbildungsmaßnahmen ausmachen (Hübler 2003, S. 391).

Dass die vorliegenden Untersuchungen z. T. kontoverse Ergebnisse erkennen lassen, muss zunächst einmal kein Nachteil sein. Ziel der Forschung kann nicht ein schlichtes Pro oder Kontra bezogen auf die deutsche Mitbestimmung sein. Diese ist allseits anerkannt - ein Kernelement des deutschen Produktions- und damit Innovationssystems. Vielmehr handelt es sich darum, seine Stärken und Schwächen zu erkennen. Hierzu haben die Forschungsergebnisse Erkenntnisse geliefert. Allerdings besteht angesichts einer Vielzahl offener Fragen nach wie vor ein erheblicher Bedarf an quantitativ wie qualitativ orientierter Forschung.

\subsection{HERAUSFORDERUNGEN}

Unbestritten ist, dass sich der Druck auf die Mitbestimmung infolge der Globalisierung in den letzten Jahrzehnten enorm erhöht hat. Der Hightechstandort Deutschland kann seinen Vorsprung in den Schlüsselbranchen, aber auch in neu entstehenden Branchen, nur bei Aufrechterhaltung von Innovationsvorsprüngen bewahren. Hier hat sich zweifellos die Funktion von Mitbestimmung gewandelt. Sie hat nicht mehr allein die Aufgabe, durch eine Zivilisierung des Rationalisierungsprozesses den beruflichen Status und die Arbeitsplätze zu sichern und, wenn möglich, für ,gute Arbeit" zu sorgen (Kern 1998). Das bleibt notwendig, wird aber in Zukunft nicht mehr ausreichen; neue Anforderungen an ein Co-Management der Mitbestimmungsträger entstehen. In den vielen Unternehmen, die unter der Krise leiden und in ihrem Bestand gefährdet sind, sind Akteure der Mitbestimmung aktuell gefordert, eigenständige Beiträge zum Gelingen von Innovationen zu leisten, um ihre Arbeitsplätze zu sichern. Dies ist bereits teilweise betriebliche Praxis (vgl. Schwarz-Kocher et al. in diesem Heft), aber doch nur eine Facette des Problems. Generell geht es um einen stärkeren Beitrag der Mitbestimmungsinstitutionen bei der Aktivierung des Wissens der Beschäftigten für betriebliche Innovationsprozesse. Durch die Mitbestimmung kann die Kooperationsbereitschaft - wie die vorliegenden Forschungsergebnisse zeigen - erhöht werden. Die Chancen für eine Ausschöpfung der internen Ressourcen und Kompetenzen eines
Unternehmens verbessern sich, wodurch die Komplexität von Innovationsprozessen mit ihren Rückkoppelungen besser handhabbar wird.

Noch sind solche Zielvorstellungen nicht voll realisiert. Die Gewerkschaften plädieren für ein stärkeres Engagement der Mitbestimmungsträger im Innovationsprozess, beispielsweise strebt die IG Metall mit der Kampagne „Besser statt billiger“ einen offensiveren Umgang mit betrieblichen Innovationen an, um die Beschäftigung durch eine verbesserte Wettbewerbsfähigkeit zu sichern (Korflür et al. in diesem Heft). Damit wendet sich diese Kampagne explizit gegen Strategien der Unternehmen, die sich häufig auf schlichte Kostensenkung zum Nachteil der Beschäftigten beschränken. Über die betriebliche Ebene hinaus sollen die betrieblichen Initiativen gewissermaßen zu einer Branchenpolitik bzw. Industriepolitik gebündelt werden. Dem liegt ein umfassendes Verständnis von Innovationsprozessen zugrunde, das die Aporien eines auf die technischen Dimensionen verkürzten Innovationsbegriffes vermeidet, denn „Innovation ist mehr als Technik“ (Klotz 2003).

Ihre Interessenlage sowie ihr arbeitsorientiertes Verständnis prädestiniert die Mitbestimmungsträger (und die Gewerkschaften) für die Einbeziehung der Dimensionen von betrieblichen Innovationen, die im betrieblichen Alltag häufig zu kurz kommen. Sie können damit zugleich eine breitere Beteiligung der Beschäftigten fördern, die der betrieblichen Innovationsfähigkeit zugute kommen würde (Kirner et al. in diesem Heft). Die vorliegenden Forschungsergebnisse zeigen - bei aller Differenz -, dass für das Gelingen von Innovationen wichtige Faktoren von den Betriebsräten (mit)bestimmt werden: die Arbeitsorganisation, die Qualifizierung und die Akzeptanz in der Belegschaft. Sie können einen Beitrag für eine innovationsfreundliche Atmosphäre im Unternehmen leisten. Innovationen sind ja häufig nicht planbar, sie entstehen nicht selten an den „Rändern“ einer Organisation. Dieses gilt insbesondere für die Planung von Produktinnovationen. $\mathrm{Ob}$ sie erfolgreich sind, ist nicht vorhersehbar, sondern erweist sich erst im Prozess der Innovation selbst. Damit sie eine Chance haben, sich in der betrieblichen Hierarchie „durchzusetzen“, bedarf es entsprechender Rahmenbedingungen, d. h. Freiräume sowie einer vertrauensvollen, ermunternden Umgebung.
Betriebsräte sind hier als Promotoren gefordert, um sich für die entsprechenden Rahmenbedingungen einzusetzen (Sperling/Wolf in diesem Heft).

Freilich, es wäre naiv anzunehmen, dass eine erweiterte und offensive Innovationspolitik sich ohne Probleme umsetzen ließe. Trotz verbesserten Mitbestimmungsmöglichkeiten sowie eines von den Märkten kommenden spürbaren Handlungsdrucks steht bei vielen Betriebsräten das Thema Innovation nicht im Zentrum ihrer Politik (Kriegesmann et al. in diesem Heft). Die klassischen sozialen und personalen Aufgaben dominieren noch immer. Zudem ist mit Widerständen vonseiten des Managements zu rechnen. Es wird oft zum Schaden des Innovationsprozesses durch Macht schlicht dekretiert (Scholl 2004). ${ }^{6}$ Es handelt sich also keineswegs um ein konfliktfreies Feld, auf dem beide Seiten immer automatisch ihren jeweiligen Nutzen erzielen. Beschäftigungs- bzw. Innovationspakte im Sinne eines do ut des sind erforderlich, aber nicht leicht zu erreichen (Schwarz-Kocher et al. in diesem Heft).

\section{Fazit und Ausblick}

In der aktuellen tief greifenden Krise ist mit dramatischen Strukturveränderungen sowie einer Verschiebung der Gewichte in der Weltwirtschaft zu rechnen. Dies zeigt sich jetzt schon, z. B. im Maschinenbau, einer deutschen Kernbranche (Dispan 2009). Es ist keineswegs ausgemacht, dass die deutsche Volkswirtschaft gestärkt aus dieser Krise herauskommen wird. Dieses wird u. a. davon abhängen, inwieweit es gelingt, ihre Innovationsfähigkeit zu erhöhen. Keineswegs reicht hierfür ein höherer Input von Mitteln für Forschung und Entwicklung aus - so notwendig er auch ist. Genauso wichtig ist es, die Stärken des deutschen Entwicklungspfades zu nutzen und seine Schwächen zu vermeiden. Die Mitbestimmung als Kernelement des deutschen Produktionssystems ist hier in dem Sinne gefordert, dass die Kompetenzen im Betrieb und im Unternehmen stärker aus-

\footnotetext{
6 Das von W. Scholl geleitete und von der HansBöckler-Stiftung geförderte Projekt „Innovativität durch Mitbestimmung, untersucht an der Beteiligung von Betriebsräten an Prozessinnovationen “ befasst sich mit dieser Problematik.
} 
geschöpft werden. „Offene Innovation“ (Reichwald/Piller 2009) impliziert eben auch die Öffnung des Innovationsprozesses nach innen. Wie erreicht man eine solche Stärkung des deutschen Entwicklungspfades? Um das Verhältnis von Mitbestimmung und Innovation mit seinen Stärken und Schwächen genauer auszuleuchten, hat die Hans-Böckler-Stiftung mehrere Forschungsprojekte vergeben. Ihre teils vorläufigen Ergebnisse werden in diesem Schwerpunktheft präsentiert. Ausgangspunkt ist die Einschätzung, dass Verbesserungen der Funktionalität der Mitbestimmung für Innovationsprozesse im Prinzip anzustreben sind. Wissenschaftlich gut dokumentierte Beispiele können hier hilf- reich sein. Die Chancen für einen solchen forschungspolitischen Ansatz stehen nicht schlecht. Die häufig neoliberal unterfütterte Kritik an der Mitbestimmung als Innovationsbremse erweist sich beim jetzigen Stand der Forschung als wenig tragfähig und ist angesichts des Debakels eines entfesselten Finanzkapitalismus generell etwas zurückhaltender geworden.

Die Beiträge in diesem Heft konzentrieren sich auf die Ebene des Unternehmens bzw. Betriebes als dem Ort, an dem Innovationen maßgeblich entwickelt bzw. umgesetzt werden. Nicht minder wichtig sind jedoch die Branchen, der Staat sowie die Europäische Union - werden doch dort innovationspolitische Rahmenbedingun- gen bereitgestellt. Hier können wir immer noch eine Reduzierung des Innovationsverständnisses auf technologische Aspekte, insbesondere innerhalb von HightechStrategien, konstatieren. Wir wissen heute, dass ein solcher Ansatz zu kurz greift. Es ist zu hoffen, dass bei der anstehenden PostLissabon-Strategie (Kommission der Europäischen Gemeinschaften 2009) und der Weiterentwicklung der Hightech-Strategie der Bundesregierung die Träger der Mitbestimmung ihr Gewicht in die Waagschale werfen können, um der forschungspolitischen Umsetzung eines ganzheitlichen Innovationsansatzes zum Durchbruch zu verhelfen.

\section{LITERATUR}

Addison, J. T./Schnabel, C./Wagner, J. (1996): German Works Councils, Profits and Innovation, in: Kyklos 49, Basel, S. 555-582

Addison, J. T./Schnabel, C./Wagner, J. (2001): Works Councils in Germany: Their Effects on Firm Performance; in: Oxford Economic Papers 53, Oxford

Alchian, A. A. (1984): Specifity, Specialisation and Coalitions, in: Journal of Institutional and Theoretical Economics 140, Tübingen, S. 54-70

Akerloff, G. A./Shiller, R. J. (2009): animal spirits - Wie Wirtschaft wirklich funktioniert, Frankfurt/NewYork

Arreger, K. (1976): Innovation in sozialen Systemen, Bern/Stuttgart Askildson, J. E./Jirjahn, U./Smith, S. C. (2002): Works Councils and Environmental Investment. Theory and Evidence from German Panel Data, in: CESifo Working Paper 785, München

Bluestone, B./Harrision, B. (1982): The Deindustrialisation of America, New York

Blume, L./Gerstlberger, W. (2007): Determinanten betrieblicher Innovation: Partizipation von Beschäftigten als vernachlässigter Einflussfaktor, in: Industrielle Beziehungen 3, Mering

Bundesministerium für Bildung und Forschung (BMBF) (2006): Zur technologischen Leistungsfähigkeit Deutschlands 2005, Bonn/Berlin

Bowles, S./Gordon, D. M./Weiskopf, Th. E. (1984): Beyond The Waste Land. A Democratic Alternative To Economic Decline, London

Dertouzos, M. L./Lester, R. K./Solow,R. M. (1990): Die Krise der USA, Potential für neue Produktivität "Made in USA", Frankfurt/M.

Dilger, A. (2002): Ökonomik betrieblicher Mitbestimmung. Die wirtschaftlichen Folgen von Betriebsräten, München

Dispan, J. (2009): Werkzeugmaschinenbau 2009 - Krisenwirkungen und aktuelle Herausforderungen; in: Informationsdienst des IMU-Instituts 5, Stuttgart

Dreher, C. (2008): Herausforderungen des ganzheitlichen Innovationsverständnisses an das deutsche Innovationssystem, Expertise für die Hans-Böckler-Stiftung, Karlsruhe
FitzRoy, F. R./Kraft, K. (1990): Innovation, Rent-Sharing and the Organization of Labour in the Federal Republic of Germany; in: Small Business Economics 2, New York

Furubotn, E. G. (1978): The Economic Consequences of Codetermination on the Rate and Sources of Private Investment, in: Pejovic, S. (Hrsg): The Codetermination Movement in the West. Labor Participation in the Management of Business Firms, Lexington

Gaisser, S./Nusser, M./Reiß, Th.(2005): Stärkung des Pharma-Innovationsstandortes Deutschland, ISI-Schriftenreihe "Innovationspotenziale", Karlsruhe

Gerlach, K./Jirjahn, U. (1998): Determinanten betrieblicher Weiterbildungsaktivitäten: Eine empirische Untersuchung mit Daten des Hannoveraner Firmenpanels; in: Pfeiffer, F./Pohlmeier, W. (Hrsg.): Qualifikation, Weiterbildung und Arbeitsmarkterfolg, Baden-Baden

Gerlach, F./Ziegler, A. (2007): Innovationspolitik - Wie kann Deutschland von anderen lernen?, Marburg

Gerybadze, A. (2004): Technologie- und Innovationsmanagement, München

Grupp, H. /Legler, H./Licht, G. (2004): Technologie und Qualifikation für neue Märkte. Ergänzender Bericht zur technologischen Leistungsfähigkeit Deutschlands 2003-2009, BMBF, Berlin/Bonn

Hall, P. A./Soskice, D. (2001): Varieties of Capitalism: The Institutional Foundations of Comparative Advantage, Oxford

Hübler, O. (2003): Fördern oder behindern Betriebsräte die Unternehmensentwicklung, in: Perspektiven der Wirtschaftspolitik 4, Chichester, S. 379-397

Hübler, O./Jirjahn, U. (2002): Arbeitsproduktivität, Reorganisationsmaßnahmen und Betriebsräte, in: Bellmann, L./Kölling, A. (Hrsg.): Betrieblicher Wandel und Fachkräftebedarf, BeitrAb 257, Nürnberg, S. 1-45 Jirjahn, U. (1998): Effizienzwirkungen von Erfolgsbeteiligung und Partizipation, Frankfurt/New York 
Jürgens, U./Lippert, I. (2005): Kommunikation und Wissen im Aufsichtsrat - Voraussetzungen und Kriterien guter Aufsichtsratarbeit aus der Sichtweise leitender Angestellter, WZB-Discussion Paper 2005-301, Berlin Jürgens, U./Sablowski, Th. (2008): Sektorale Innovationsprozesse und die Diskussion über deutsche Innovationsschwächen, Edition der HansBöckler-Stiftung 204, Düsseldorf

Kalmbach, P./Frank, R. /Knottenbauer, K./Krämer, H. (2005): Die Interdependenz von Industrie und Dienstleistungen. Zur Dynamik eines komplexen Beziehungsgeflechts, Berlin

Kern, H. (1998): Mitbestimmung und Innovation. Expertise für das Projekt "Mitbestimmung und neue Unternehmenskulturen“ der Bertelsmann Stiftung und der Hans-Böckler-Stiftung, Gütersloh

Klotz, U. (2003): Innovation ist mehr als Technik; in: Computer-Fachwissen 10, Düsseldorf, S. 4-11

Kommission der Europäischen Gemeinschaften (2009), Arbeitsdokument der Kommission. Konsultationen über die künftige EU-Strategie bis 2020, KOM (2009) 647 24.11.2009, Brüssel

Kraft, K./Stank, J./Dewenter, R. (2009): Co-Determination and Innovation, IZA-DiscussionPaper 4487, Bonn

Krueger, A. B./Solow, R. M. (Hrsg.) (2001): The Roaring Nineties - Can Full Employment Be Sustained?, New York

Licht, G./Rammer, Ch./Sellenthin, M. O. (2009): Indikatoren zur Innovationskraft Deutschland im internationalen Vergleich und aktuelle Entwicklungen der Innovationspolitik, Kurzexpertise im Auftrag der HansBöckler-Stiftung, Mannheim

Lundvall, B. A. (1992): National Systems of Innovation: Towards a Theory of Innovation and Interactive Learning, London
Nelson, R. R. (1993): National Innovations Systems: A Comparative Study, Oxford

OECD (2002): The Measurement of Scientific and Technological Activities. Proposed Standard Practise for Surveys on Research and Experimental Development, Frascati Manual, Paris

OECD (2005): Oslo Manual: Guidelines for Collecting and Interpreting Innovation Data, Paris

Reichwald, R./Piller, F. (2009): Interaktive Wertschöpfung, Wiesbaden Scholl, W. (2004): Innovation und Information. Wie in Unternehmen neues Wissen produziert wird, Göttingen

Schumpeter, J. A. (1932): Business Cycles. A Theoretical, Historical and Statistical Analysis of the Capitalist Process, New York/London

Stiglitz, J. E. (2004): Die Roaring Nineties. Der entzauberte Boom, Berlin

Stiglitz, J. E. (2009): Das Geheimnis der unsichtbaren Hand, in: Süddeutsche Zeitung vom 31.12.2009/1.1.2010

Tidd, J./Bessant, J./Pavitt, K. (2001): Managing Innovation - Integrated Technology Market and Organisational Change, Chichester

Verband Deutscher Maschinen- und Anlagenbau e. V. (VDMA) (2009): Maschinenbau in Zahlen und Bild 2009, Frankfurt am Main

Volcker, P. (2009): “Amerika muss sich ändern", in: Der Spiegel 51, vom 14.12.2009, Hamburg

Xielin, L./Lüthje, B./Pawlicki, P. (2007): China: Nationales Innovationssystem und marktwirtschaftliche Transformation, in: Gerlach, F./Ziegler, A. (Hrsg): Innovationspolitik - Wie kann Deutschland von anderen lernen?, Marburg 